\title{
Tuberculosis esofágica asociada con inmunosupresión por VIH: reporte de caso
}

\section{Esophageal tuberculosis associated with HIV immunosuppression: Case report}

Edwar Jassir Rozo-Ortiz, ${ }^{*}$ Mónica Paola Quemba-Mesa, ${ }^{2}$ @] Juan David Bustos-Acosta, ${ }^{3}$ Iván Yesid Castellanos-Pedroza, ${ }^{3}$ Jamir Muñoz-Torres, ${ }^{3}$ (i) Ledmar Jovanny Vargas-Rodríguez. ${ }^{4}$ (B)

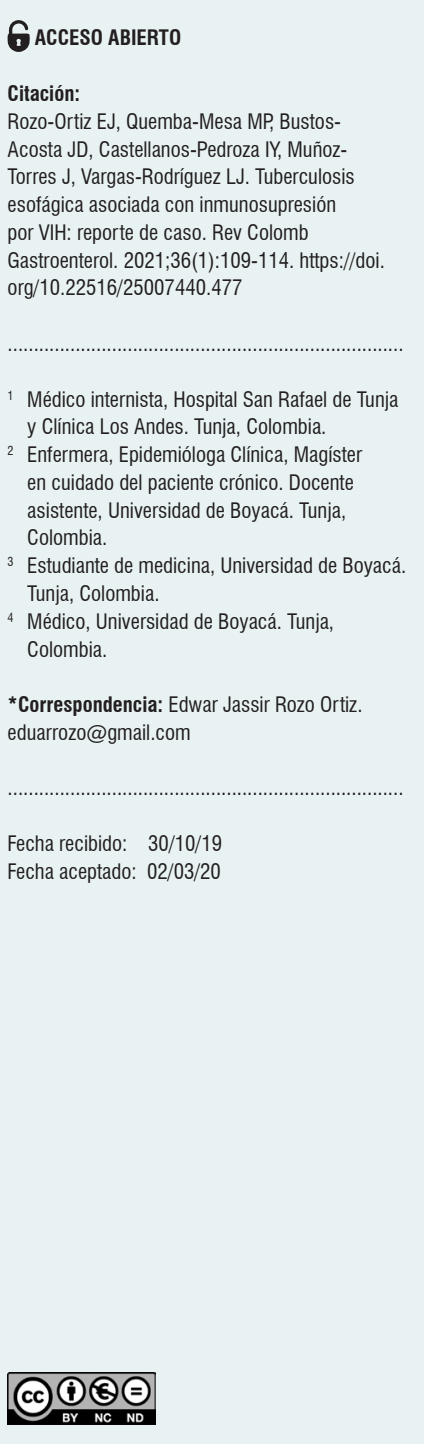

\section{Resumen}

Introducción: la tuberculosis es una enfermedad infecciosa que suele afectar a los pulmones y es causada por Mycobacterium tuberculosis. Se transmite de una persona a otra a través de gotículas generadas en el sistema respiratorio de pacientes con enfermedad pulmonar activa. La tuberculosis esofágica es una afección rara (representa solo el 2,8 \% de todos los casos de tuberculosis gastrointestinal). Por lo general, ocurre como resultado de la diseminación directa desde los ganglios mediastínicos (rara vez desde los pulmones o el torrente sanguíneo). Su diagnóstico definitivo requiere el aislamiento de los bacilos tuberculosos, lo que rara vez se logra en la práctica clínica; aun así, clínicamente los pacientes presentan síntomas de disfagia, odinofagia y adelgazamiento. Caso clínico: se presenta un paciente de 40 años de edad con cuadro clínico de 6 meses de evolución con disfagia progresiva exacerbada a disfagia para líquidos, astenia, adinamia, hiporexia, dolor pleurítico, epigastralgia y tos con expectoración blanquecina; se realizó una endoscopia de vías digestivas altas que mostró una mucosa gravemente inflamada con ulceraciones irregulares de hasta 2 $\mathrm{cm}$ de profundidad, lesiones que sugirieron tuberculosis esofágica, confirmada por el estudio histopatológico: esofagitis crónica granulomatosa; por tanto, se inició el tratamiento tetraconjugado: rifampicina, isoniazida, pirazinamida y etambutol. Conclusiones: La tuberculosis con afección del tubo digestivo es una entidad de baja frecuencia; adicionalmente, es importante recordar que la gravedad del cuadro clínico usualmente está relacionada con la coinfección por el virus de la inmunodeficiencia humana $(\mathrm{VIH})$, y que la asociación de ambas patologías es frecuente.

\section{Palabras clave}

Esófago, tuberculosis, inmunosupresión, virus de la inmunodeficiencia humana $(\mathrm{VIH})$, endoscopia, disfagia.

\begin{abstract}
Introduction: Tuberculosis is an infectious disease that usually affects the lungs and is caused by Mycobacterium tuberculosis. It is transmitted from one person to another through droplets generated in the respiratory system of patients with active lung disease. Esophageal tuberculosis is a rare condition (accounting for only $2.8 \%$ of all cases of gastrointestinal tuberculosis). It usually occurs as a result of the direct spread from the mediastinal nodes, but rarely from the lungs or bloodstream. Its definitive diagnosis requires the isolation of tuberculous bacilli, which is rarely achieved in clinical practice. However, clinically speaking, patients present with symptoms of dysphagia, odynophagia, and weight loss. Case study: This is the case of a 40 -year-old patient with symptoms of 6 months of evolution with progressive dysphagia exacerbated to dysphagia for liquids, asthenia, adynamia, hyporexia, pleuritic pain, epigastric pain, and cough with whitish sputum. An endoscopy of the upper digestive tract was performed, showing severely inflamed mucosa with irregular ulcerations up to $2 \mathrm{~cm}$ deep, which suggested esophageal TB. The diagnosis was confirmed by histopathology, which reported chronic granulomatous esophagitis. Tetraconjugated treatment was initiated, including rifampicin, isoniazid, pyrazinamide, and ethambutol. Conclusions: Gastrointestinal tuberculosis is a rare entity. It should be noted that the severity of the symptoms is usually associated with HIV co-infection, and their association is a frequent occurrence.
\end{abstract}

Keywords

Esophagus, Tuberculosis, Immunosuppression, HIV, Endoscopy, Dysphagia. 


\section{INTRODUCCIÓN}

Según datos de la Organización Mundial de la Salud (OMS), la tuberculosis continúa siendo un problema de salud pública. Para 2015, se estimaron 10,4 millones de casos nuevos de tuberculosis en todas sus formas a nivel mundial y se notificaron 6,1 millones; mientras que en 2016 se reportaron al Sistema de Vigilancia de Salud Pública colombiano (Sivigila) 13626 casos de tuberculosis en Colombia. De esta cifra, 2147 correspondieron a coinfección con el virus de la inmunodeficiencia humana (VIH) y la incidencia registrada fue de 27,9 casos por cada 100000 habitantes (1). En todo el mundo, 36,7 millones de personas vivían con el VIH a finales de 2015; 2,1 millones de personas contrajeron la infección por el VIH (finales de 2015) y 1,1 millones de personas fallecieron a causa de enfermedades relacionadas con el síndrome de inmunodeficiencia adquirida (SIDA) $(2,3)$.

La tuberculosis extrapulmonar se confirma cuando una persona presenta infección en otros órganos que no son los pulmones (pleura, ganglios linfáticos, abdomen, tracto genitourinario, piel, articulaciones, huesos, esófago o meninges) $(4,5)$. La tuberculosis esofágica es una afección rara (representa solo el 2,8\% de todos los casos de tuberculosis gastrointestinal). Por lo general, ocurre como resultado de la diseminación directa desde los ganglios mediastínicos (rara vez desde los pulmones o el torrente sanguíneo) (6). La afectación primaria de la tuberculosis en el esófago no es común debido a mecanismos protectores tales como el epitelio escamoso estratificado de múltiples capas, la mucosidad, la saliva, el peristaltismo y la postura erecta, lo que hace que la bacteria permanezca en el esófago solo de forma transitoria (7). La tuberculosis esofágica no tiene características clínicas, de laboratorio, radiológicas o endoscópicas. Su diagnóstico definitivo requiere del aislamiento de los bacilos tuberculosos, lo que rara vez se logra en la práctica clínica (8); aun así, clínicamente los pacientes presentan síntomas de disfagia, odinofagia y adelgazamiento. Estos hallazgos clínicos junto con los endoscópicos pueden mimetizar un diagnóstico de carcinoma del esófago (9). En el presente estudio se reporta un paciente con presencia de tuberculosis esofágica asociada con inmunosupresión por VIH.

\section{PRESENTACIÓN DEL CASO}

Se trata de un varón de 40 años edad con antecedentes de consumo crónico de guarapo durante aproximadamente 30 años y VIH diagnosticado una semana antes. Ingresó al servicio de urgencias por un cuadro clínico de 6 meses de evolución consistente en la pérdida progresiva de peso (aproximadamente $40 \mathrm{~kg}$ ), asociado con disfagia progresiva exacerbada a disfagia para líquidos, astenia, adinamia, hiporexia, dolor pleurítico, deposiciones sanguinolentas y epigastralgia desde hace 10 días; además, refirió episodios de tos con expectoración blanquecina.

$\mathrm{Al}$ examen físico de ingreso a urgencias presentó los siguientes signos vitales: presión arterial (PA): 80/60 mm $\mathrm{Hg}$, presión arterial media (PAM): $80 \mathrm{~mm} \mathrm{Hg}$, frecuencia cardíaca (FC): 90 latidos por minuto (lpm), frecuencia respiratoria (FR): 22 respiraciones por minuto (rpm), temperatura $\left(\mathrm{T}^{\circ}\right): 36,5$, saturación de oxígeno $\left(\mathrm{SatO}_{2}\right): 93 \%$. Se encontraba alerta, con actitud distante, hostil, hipoproséxico, con ideas de minusvalía y desesperanza, afecto hipotímico de fondo irritable, introspección y prospección parcial, juicio debilitado, tendencias e ideas de suicidio, caquéctico, con mucosa oral con placas blanquecinas en paladar duro y carrillos, enoftalmia, abdomen distendido y doloroso a la palpación en el hipocondrio izquierdo, sin signos de irritación peritoneal, extremidades con marcada atrofia muscular, desnutrición proteicocalórica, piel brillante y descamativa.

Como apoyo diagnóstico, se realizó baciloscopia que dio positivo y endoscopia de vías digestivas altas, en la que se reportaba mucosa gravemente inflamada con ulceraciones irregulares de hasta $2 \mathrm{~cm}$ de profundidad, sucias recubiertas de fibrina, con imágenes de lesiones elevadas irregulares de hasta $1 \mathrm{~cm}$ de extensión, sugestiva de tuberculosis gastrointestinal; lo anterior fue confirmado por el estudio histopatológico: esofagitis crónica granulomatosa sugestiva de infección por tuberculosis (Figura 1) y tomografía axial computarizada (TAC) torácica que revela nódulos centrolobulillares con opacidad de árbol en gemación, cuyas características sugieren micobacterias atípicas o típicas, y lesión ocupante de espacio de aspecto infiltrativo a nivel del lóbulo superior derecho (Figura 2). Adicionalmente, en el cuadro hemático se encontró leucocitos $5040 / \mathrm{mm}^{3}$, neutrófilos: $70 \%$, linfocitos: $19 \%$, hemoglobina 9,6 : \%, plaquetas: $271000 / \mathrm{mm}^{3}$, tiempo de protrombina: 18 segundos, índice internacional normalizado (INR): 1,45, nitrógeno ureico sanguíneo (BUN): $12 \mathrm{mg} / \mathrm{dL}$, creatinina: $0,6 \mathrm{mg} /$ $\mathrm{dL}$, aspartato-aminotransferasa (AST): $123 \mathrm{UI} / \mathrm{L}$, alaninaaminotransferasa (ALT): $44 \mathrm{UI} / \mathrm{L}$, lactato-deshidrogenasa (LDH): 129 UI/L, PCR: 2,07; pruebas complementarias: VIH 191 (positivo), VHC (no reactivo), VHB 0,4 (negativo), treponema (negativo), toxoplasma inmunoglobulina $M(\operatorname{IgM})$ 0,2 (negativo).

Ante estos resultados se dio el manejo con rifampicina $(150 \mathrm{mg})+$ isoniazida $(75 \mathrm{mg})+$ pirazinamida $(400 \mathrm{mg})$ + etambutol ( $275 \mathrm{mg}$ ) cada 24 horas; sin embargo, al sexto día de iniciado el tratamiento el paciente presentó deterioro sistémico y falleció. 

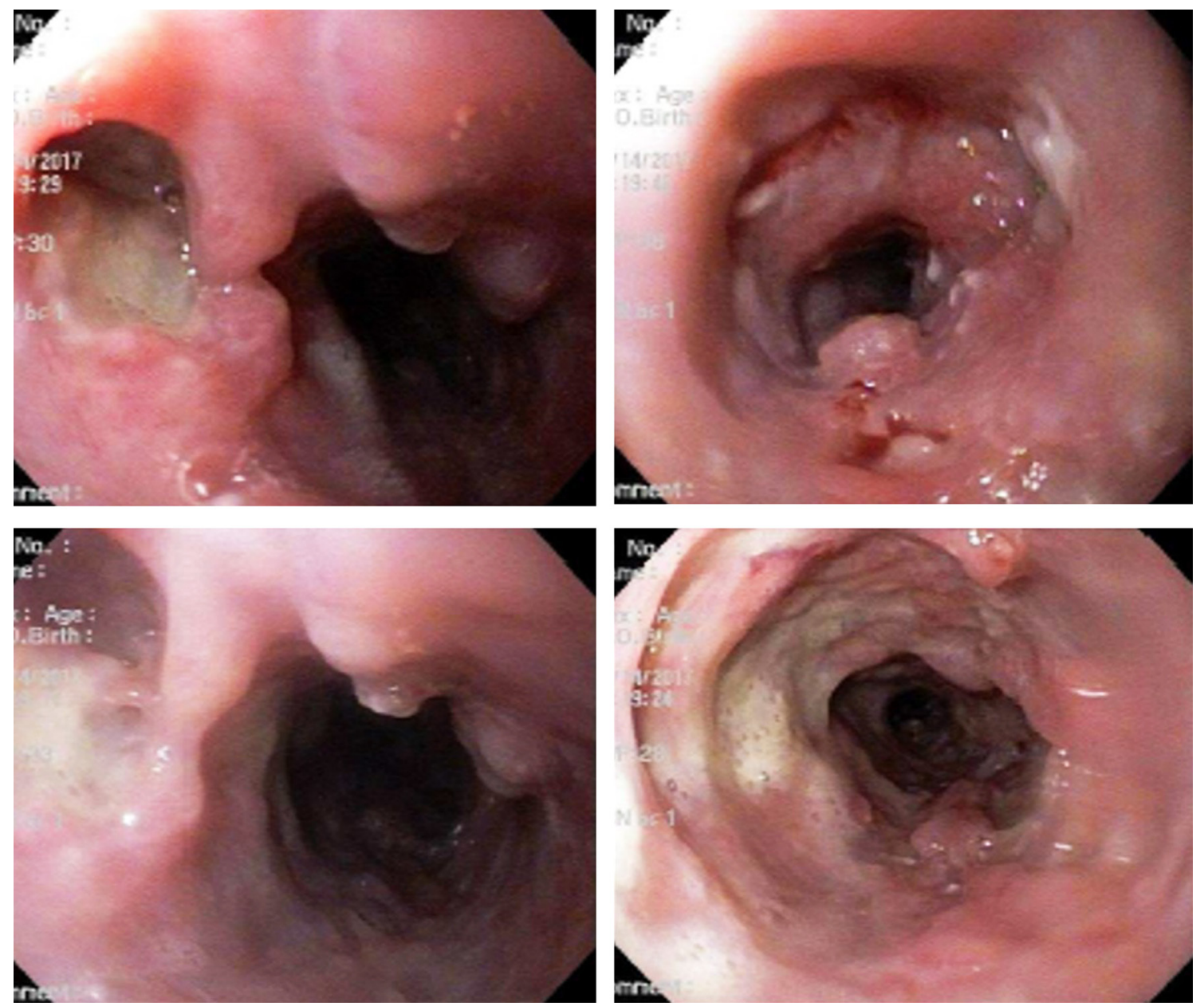

Figura 1. Los hallazgos sugieren tuberculosis gastrointestinal.

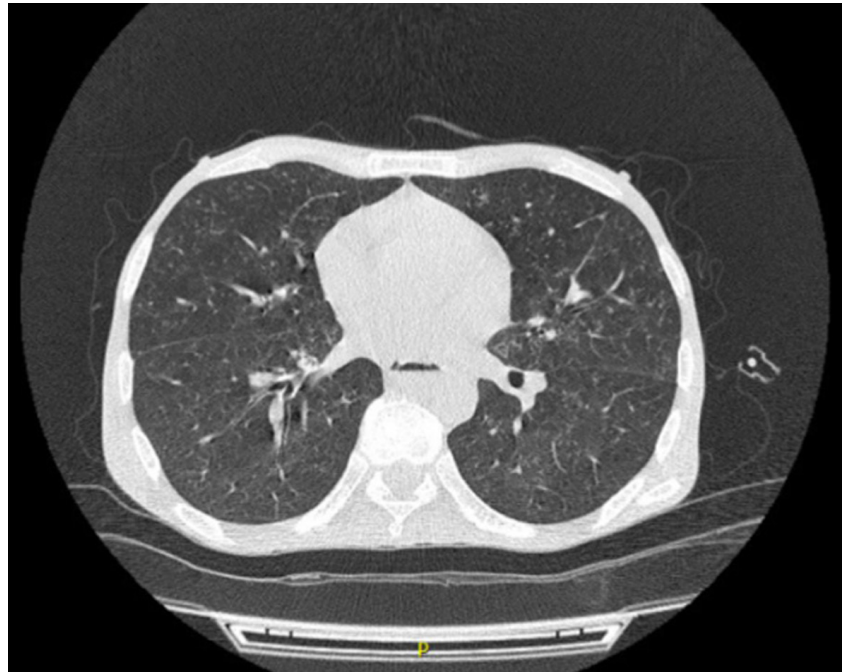

Figura 2. Nódulos centrolobulillares con opacidad de árbol en gemación.

\section{DISCUSIÓN}

En relación con las manifestaciones clínicas, del total de pacientes que cursan con tuberculosis, $10 \%-20 \%$ corresponde a localizaciones extrapulmonares, dicho porcentaje aumenta de manera considerablemente (45\%-75\%) en pacientes con infección por el VIH (10). La base de la alta frecuencia de tuberculosis extrapulmonar entre pacientes infectados por VIH es la falla de la respuesta inmunitaria contra Mycobacterium tuberculosis, lo que permite la diseminación hematógena y la subsiguiente mono- o poliinvasión de tejidos extrapulmonares.

La presentación clínica de la tuberculosis esofágica es aún indeterminada, se puede mostrar como un espectro de características clínicas difusas; sin embargo, buena parte de los casos reportados en la literatura científica confluyen en una típica presentación de disfagia y odinofagia, con disminución 
del apetito y pérdida de peso involuntaria, según los casos reportados por Garg R, Carter S, 2017 (11) y Martins M y colaboradores, 2016 (9). Aun así, la disposición de dichas manifestaciones no siempre es bien definida, lo que da lugar a erróneos diagnósticos, como puede ser la presentación acompañada de dolor retroesternal, epigastralgia y vómito, según Kumar y colaboradores, 2016 (12). Debido a la localización gastrointestinal de la tuberculosis esofágica, las manifestaciones relacionadas con dicho sistema son frecuentes, aunque inespecíficas para su detección y diagnóstico, como pueden ser la secreción purulenta anorrectal, según Zuleta y colaboradores, 2012 (13), y hematemesis, conforme lo reportado por Manrique y colaboradores, 2014 (14).

Frente a los paraclínicos, en términos generales, realizar el diagnóstico de tuberculosis extrapulmonar es difícil debido a que es una patología poco común y existe poca familiaridad con su presentación clínica; además de la inaccesibilidad a los lugares de infección y la baja carga bacteriana, que dificulta más el diagnóstico (10). La endoscopia digestiva alta con biopsia es la herramienta diagnóstica más útil, a pesar de que no existen hallazgos característicos y muchas veces la anatomía patológica no es concluyente (9).

Habitualmente, la endoscopia de vías digestivas altas muestra una úlcera solitaria de base cavada y bordes elevados ubicados a nivel del tercio medio del esófago $(9,15)$; sin embargo, como se demostró anteriormente, no se presenta una lesión clásica o típica de la patología. La literatura científica establece que los hallazgos endoscópicos esofágicos varían desde úlceras superficiales hasta lesiones elevadas que hacen pensar en lesiones neoplásicas, así como estenosis y fistulas $(16,17)$. En contraste, Hun Seo y colaboradores establecieron que los hallazgos endoscópicos de la tuberculosis esofágica incluyen tipos ulcerativos, granulares o hipertrofiados; y el hallazgo más común es la ulceración lineal con márgenes irregulares y una base grisácea con un borde infiltrado irregular en el esófago medio (7).

No obstante, Laverdure y colaboradores reportaron el caso de un paciente con el siguiente hallazgo endoscópico: lesión que genera el estrechamiento del lumen, con una depresión longitudinal en su centro y sin evidencia de ulceración o inflamación de la mucosa (18); en contraste con nuestro caso, que reporta ulceraciones irregulares de hasta $1 \mathrm{~cm}$ en toda su extensión acompañado de reacción inflamatoria grave y reducción de la luz esofágica, lesiones muy similares a las reportadas por Lagos y colaboradores (19), y Momin y colaboradores (20). El diagnóstico definitivo se logra a través del aislamiento de bacilos en los medios de cultivo apropiado $(9,10)$, la presencia de granulomas caseificantes y de bacilos ácido-alcohol resistentes en las biopsias $(9,15,17,20,21)$. Con respecto al diagnóstico endoscópico, reportamos una esofagitis crónica granulomatosa sugestiva de infección por tuberculosis, compatible con el reporte de Bresky y colaboradores (10), Mohan y colaboradores (22) y Manrique y colaboradores (14).

Pese a lo manifestado, Momin y colaboradores, y Zuleta y colaboradores reportaron casos con histopatología normal, sin presencia de granulomas caseificantes y ni de bacilos ácido-alcohol resistentes $(20,21)$. Las características histológicas tienen una amplia gama de patrones como la intensa infiltración de linfocitos, la marcada reacción inflamatoria (23) o la presencia de células gigantes del tipo Langhans (12, 19, 24). Finalmente, para el diagnóstico definitivo, además del estudio histopatológico se usa la detección de fragmentos del genoma bacilar por técnicas de PCR, las cuales han mostrado una sensibilidad del $80 \%$ y una especificidad del $100 \%$ en el diagnóstico de tuberculosis extrapulmonar (9).

Adicionalmente, una prueba de alto impacto actual es el uso de la endosonografía, también conocida como ecografía endoscópica, la cual tiene hallazgos característicos de esta patología; uno de estos es la presencia de ganglios linfáticos adyacentes a la patología esofágica, de localización subcarinal o periesofágica, los cuales se encontraban enmarañados $(8,25)$, heterogéneos con una ecotextura predominantemente hipoecogénica (26) o anecoica, particularmente en el centro y algunos hilos y focos (manchas) hiperecogéni$\cos (7,8,25)$. Otros hallazgos típicos son el engrosamiento de la pared esofágica y la formación de masas con disrupción de la adventicia $(7,27)$.

En relación con el tratamiento, el régimen farmacológico estándar recomendado para la tuberculosis esofágica corresponde a tratamiento antituberculoso tetraconjugado (rifampicina, isoniazida, pirazinamida, etambutol $)(10,13,14,22)$ por 2 meses más isoniacida y rifampicina por 4 meses adicionales (14); sin embargo, se puede usar estreptomicina por etambutol (23). Algunos autores afirman el uso de esquema triple (21): isoniacida, rifampicina y pirazinamida (17), y algunos deciden adicionar piridoxina (9). Generalmente, estos pacientes tienen buen pronóstico si se hace un diagnóstico temprano $(21,23)$ con tasas del éxito del $90 \%(14)$; pero en pacientes inmunosuprimidos, particularmente los pacientes con SIDA $(17,21,23)$, no responde adecuadamente el tratamiento antituberculoso y pueden tener una evolución fatal debido a la agresividad de la infección $(21,23)$. Por esta razón, se establece que la eficacia del tratamiento depende del estado del sistema inmunitario del paciente (13). A pesar de que el paciente del caso reportado recibió el tratamiento farmacológico oportuno (rifampicina $150 \mathrm{mg}+$ isoniazida $75 \mathrm{mg}+$ pirazinamida 400 $\mathrm{mg}$ + etambutol $275 \mathrm{mg}$ ), tuvo un deterioro sistémico progresivo de su estado de salud que lo llevó a la muerte.

\section{CONCLUSIÓN}

La tuberculosis esofágica es una entidad poco frecuente de la tuberculosis extrapulmonar, A pesar del amplio espectro 
de manifestaciones clínicas, la disfagia es una de las más representativas. La coinfección con VIH ha sido frecuente en los últimos años. El uso oportuno de la endoscopia de vías digestivas altas y la ecografía esofágica son de gran ayuda para orientar el diagnóstico, que se logra confirmar por medio del estudio histopatológico de las lesiones.

\section{Contribución al manuscrito}

Asesoría y construcción desde el ámbito clínico: Edwar Jassir Rozo-Ortiz. Asesoría y construcción desde el ámbito metodológico: Mónica Paola Quemba-Mesa. Recolección de trabajo de campo y construcción de reporte de caso: demás autores.

\section{Conflictos de interés}

Los autores declaran no presentar conflictos de intereses.

\section{Fuentes de financiación}

Se recibió financiación por parte de la Universidad de Boyacá.

\section{REFERENCIAS}

1. Fadul-Pérez S. Protocolo de Vigilancia en Salud Pública, tuberculosis, código: 813. INS; 2020 [consultado el 30 de enero de 2021]. Disponible en: https: / www.ins.gov.co/ buscador-eventos/Lineamientos/PRO_Tuberculosis.pdf

2. Onusida. Hoja informativa - Últimas estadísticas sobre el estado de la epidemia de sida. [consultado el 30 de mayo de 2018]. Disponible en: http://www.unaids.org/es/resources/fact-sheet

3. OMS. VIH/sida. [consultado el 30 de mayo de 2018]. Disponible en: https://www.who.int/es/news-room/factsheets/detail/hiv-aids

4. Tang N, Frank A, Pahalawatta V, Lampinen J, CoblenzKorte A, Dunn C, Li C, Cloherty G, Abravaya K, Leckie G. Analytical and clinical performance of Abbott RealTime MTB, an assay for detection of Mycobacterium tuberculosis in pulmonary specimens. Tuberculosis (Edinb). 2015;95(5):613-9. https://doi.org/10.1016/j. tube.2015.05.010

5. Procedimientos para la prevención y control de la tuberculosis. Manual 2017. Quito: Ministerio de Salud Pública de Ecuador; 2017 [consultado el 30 de mayo de 2018]. Disponible en: https://www.salud.gob.ec/wp-content/ uploads/2017/07/MANUAL-DE-PROCEDIMIENTOSDE-TB-FINAL.pdf

6. Khanna V, Kumar A, Alexander N, Surendran P. A case report on esophageal tuberculosis - A rare entity. Int J Surg Case Rep. 2017;35:41-43. https://doi.org/10.1016/j. ijscr.2017.03.042

7. Seo JH, Kim GH, Jhi JH, Park YJ, Jang YS, Lee BE, Song GA. Endosonographic features of esophageal tuberculosis presenting as a subepithelial lesion. J Dig Dis. 2017;18(3):185-188. https://doi.org/10.1111/17512980.12454

8. Tang Y, Shi W, Sun X, Xi W. Endoscopic ultrasound in diagnosis of esophageal tuberculosis: 10-year experience at a tertiary care center. Dis Esophagus. 2017;30(8):1-6. https://doi.org/10.1093/dote/dox031

9. Martins M, Juliana O, Carvalho A, Santos A, Santos-Costa C, Monteiro J, Abreu A, Esteves J, Garcia J, Pinto-Correia
J. Tuberculose esofágica primária: desafio para o cirurgião. Rev Port Cir. 2016;(38):31-35.

10. Bresky G, Silva N, Madariaga J. Esofagitis tuberculosa en un paciente HIV positivo. Rev Méd Chile. 2007;135(10):1323-1326. http://dx.doi.org/10.4067/ S0034-98872007001000014

11. Garg R, Carter S, Barawi M. Dysphagia from esophageal tuberculosis in a patient with undiagnosed HIV infection. Endoscopy. 2017;49(8):E195-E196. https://doi. org/10.1055/s-0043-110667

12. Kumar S, Minz M, Sinha SK, Vaiphei K, Sharma A, Singh S, Kenwar DB. Esophageal tuberculosis with coexisting opportunistic infections in a renal allograft transplant recipient. Transpl Infect Dis. 2017;19(1). https://doi. org/10.1111/tid.12640

13. Zuleta MA, Viveros D, Cañón DP. Tuberculosis intestinal: reporte de caso y revisión de la literatura. Infectio. 2012;16(3):178-182. https://doi.org/10.1016/S01239392(12)70009-7

14. Manrique CA, Salinas DC, Martínez JD, Garzón OM, Hernández CG, Hormaza N, Lizarazo J, Marulanda J, Molano JC, Rey M. Tuberculosis esofágica: informe de un caso. Rev Col Gastroenterol. 2014;29(4):446-448.

15. Sala A, Leibovich N, Souto G, Sabatini C, Brodersen C, Segal E. Tuberculosis esofágica: presentación de un caso y revisión de la literatura. Acta Gastroenterol Latinoam. 2011;41(1):47-51.

16. Salinas DC, Moreno DC, Dennis RJ. Tuberculosis pulmonar, esofágica y laríngea. Acta Médica Colombiana. 2014;39(1):85-9.

17. Baños R, Serrano A, Alberca F, Alajarín M, Albaladejo A, Vargas A, Molina J. Tuberculosis esofágica. Rev Gastroenterol Perú. 2006;26:200-202.

18. Laverdure N, Lepilliez V, Mion F. Esophageal tuberculosis: A rather specific endoscopic lesion? Clin Res Hepatol Gastroenterol. 2016;40(6):e69-e70. https://doi. org/10.1016/j.clinre.2016.05.009

19. Lagos AC, Marques I, Reis J, Esteves P, Costeiro J, Afonso A, Costa B. Tuberculose primária do esófago. GE 
J Port Gastrenterol. 2012;19(3):143-145. https://doi. org/10.1016/j.jpg.2010.08.001

20. Momin RN, Chong VH. Oesophageal tuberculosis: rare but not to be forgotten. Singapore Med J. 2012;53(9):e192-4.

21. Pioner SR, Rech D; Menegotto L, Pioner F, Santos C. Tuberculose esofágica primária - relato de caso. Rev Col Bras Cir. 2007;34(5):357-358.

22. Mohan P, Myilsamy S, Leelakrishnan V. Esophageal tuberculosis. Clin Res Hepatol Gastroenterol. 2012;36(4):3123. https://doi.org/10.1016/j.clinre.2011.12.017

23. Perdomo JA, Naomoto Y, Haisa M, Yamatsuji T, Kamikawa Y, Tanaka N. Tuberculosis of the esophagus. Dis Esophagus. 1998;11(1):72-4.

24. Montoya M, Chumbiraico R, Ricalde M, Cazorla E, Hernandez G. Blastomicosis Oral, Papilomatosis Laríngea y Tuberculosis Esofágica. Rev Peru Med Exp Salud Pública. 2012;29(2):250-254.

25. Puri R, Khaliq A, Kumar M, Sud R, Vasdev N. Esophageal tuberculosis: role of endoscopic ultrasound in diagnosis. Dis Esophagus. 2012;25(2):102-6. https://doi. org/10.1111/j.1442-2050.2011.01223.x

26. Li YX, Nian WD, Wang HH. A case of esophageal tuberculosis with unusual endoscopic feature. Clin Res Hepatol Gastroenterol. 2018;42(1):e5-e6. https://doi. org/10.1016/j.clinre.2017.04.010

27. Mou Y, Zeng H, Wang QM, Yi H, Liu W, Wen D, Hu B. Esophageal tuberculosis initially misdiagnosed by endoscopy as a submucosal tumor. Endoscopy. 2015;47 Suppl 1 UCTN:E30-1. https://doi.org/10.1055/s-0034-1391133 